\title{
$k$-means on Positive Definite Matrices, and an Application to Clustering in Radar Image Sequences
}

\author{
Daniel Fryer*, Hien Nguyen ${ }^{\dagger}$ and Pascal Castellazzi ${ }^{\ddagger}$ \\ * School of Mathematics and Physics \\ The University of Queensland, St Lucia, Australia \\ ORCID 0000-0001-6032-0522 \\ ${ }^{\dagger}$ Department of Mathematics and Statistics \\ La Trobe University, Bundoora, Australia \\ ORCID 0000-0002-9958-432X \\ $\ddagger$ Deep Earth Imaging FSP, Land and Water \\ Commonwealth Scientific and Industrial Research Organisation (CSIRO), Urrbrae, SA, Australia \\ ORCID: 0000-0002-5591-0867
}

\begin{abstract}
We state theoretical properties for $k$-means clustering of Symmetric Positive Definite (SPD) matrices, in a non-Euclidean space, that provides a natural and favourable representation of these data. We then provide a novel application for this method, to time-series clustering of pixels in a sequence of Synthetic Aperture Radar images, via their finite-lag autocovariance matrices.
\end{abstract}

Index Terms - $k$-means, Cholesky decomposition, symmetric positive definite matrices, Riemannian geometry, synthetic aperture radar, groundwater dependent ecosystems.

\section{INTRODUCTION}

Many objects of interest in applied mathematics and engineering can be represented, often uniquely, by a Symmetric Positive Definite (SPD) matrix. For example, SPD matrices correspond bijectively to mean centered Gaussian distributions, and are used to model Brownian motion in Diffusion Tensor Imaging (DTI), where they are referred to as tensors [1]. The finite-lag autocovariance matrices of time-series are SPD, and have been used in compression based clustering [2], for analysing dynamical brain functional connectivity [3], and in our application (Section III). Many more examples are mentioned in [1], [4].

For a given $m$, the space of $m \times m$ SPD matrices forms the interior of a blunt convex cone in $\mathbb{R}^{m(m+1) / 2}$, and is not a vector space under addition and scalar multiplication. Thus, many standard algorithms applied to SPD matrices, with the Euclidean norm, may produce symmetric matrices that are not positive definite, having non-positive eigenvalues [1]. Efforts to avoid this shortcoming have led to multiple suggestions for alternative Riemannian metrics, that may endow the space with a more favourable structure. The most popular of these to date has been the affine-invariant metric [5], also known in statistics as the Fisher-Rao metric [6]. Unfortunately, there is no closed form for the Fréchet mean under this metric, though an MM algorithm implementation is given in [7]. The log-Euclidean metric [8], [9] arose from attempts to put a Lie group structure on SPD matrices, thus transferring across the vector space structure of symmetric matrices, while preserving many affine-invariant qualities [1]. Recently, in [4], a new metric was introduced: the log-Cholesky metric, based on the Cholesky decomposition, a diffeomorphism between upper triangular positive definite and SPD matrices. In this work, we focus on $k$-means clustering of SPD matrices on this metric space.

Clustering via $k$-means can be used to extract information regarding heterogeneity of matrix variate data in a computationally efficient manner. Standard Euclidean $k$-means clustering, minimising within-cluster variation, is uncomplicated, gives asymptotically normal [10] and strongly consistent [11] cluster centers, and can be scaled to massive and distributed data [12]. However, performing $k$-means on SPD matrices may be difficult, without a computationally efficient form for the Fréchet mean [13].

In Section [II, we introduce the log-Cholesky distance and closed-form expression for the corresponding Fréchet mean. We then identify a diffeomorphism under which the logCholesky metric norm reduces to the Euclidean norm in $\mathbb{R}^{m(m+1) / 2}$. We then use this fact to prove that $k$-means on the log-Cholesky manifold satisfies the same consistency and asymptotic normality properties as Euclidean $k$-means. Also, we show that the average objective function converges towards its optimal value, almost surely, at a rate of $(\log (n) / n)^{1 / 2}$.

In Section III in a demonstrative application of these results, we perform $k$-means time-series clustering via finite lag autocovariance matrices, representing pixels in a sequence of Synthetic Aperture Radar (SAR) images of the Mount Gambier region of South Australia. This is done efficiently by leveraging existing low-level software libraries for computing Euclidean $k$-means, over a sample of 2,929,052 time-series, with multiple passes for parameter tuning. This results in suggestions for improvement on previous work of [14], predicting the locations of Groundwater Dependent Vegetation (GDV). A brief discussion is provided in Section IV. 


\section{A. Definition of k-means and Fréchet mean}

A manifold is a set of points that is everywhere locally homeomorphic (or, loosely speaking, is smoothly deformable) to a subset of a Euclidean space. A Riemannian manifold is a manifold $\mathcal{M}$, that is equipped with a globally defined differential structure, to allow calculus to be performed, and a Riemannian metric $g$, so that angle and length can be defined [1]. Given a random element $Q$ with distribution $P$ on a Riemannian manifold $(\mathcal{M}, g)$, with distance function $d$, the classical generalisation of the Euclidean centre of mass [8], [15] is to define the set of Karcher means,

$$
\left\{k: k=\arg \min _{x \in \mathcal{M}} \mathbb{E} d^{2}(a, Q)\right\} \subseteq \mathcal{M},
$$

as the set of points in the manifold that minimise the dispersion

$$
\mathbb{E} d^{2}(a, Q)=\int_{\mathcal{M}} d^{2}(a, q) d P(q) .
$$

When a unique minimiser exists, it is called the Fréchet mean $\mathbb{E} Q$. If the distribution $P$ is sufficiently localised, then the existence of $\mathbb{E} Q$ is guaranteed [16]. Given a finite set $\mathcal{Z} \subseteq \mathcal{M}$ of points on the manifold, we can define the empirical Fréchet mean

$$
\bar{S}=\arg \min _{a \in \mathcal{M}} \sigma^{2}(a, \mathcal{Z}),
$$

as the minimiser of the empirical dispersion

$$
\sigma^{2}(a, \mathcal{Z})=\sum_{S \in \mathcal{Z}} d^{2}(a, S) .
$$

In $k$-means clustering, we seek to find a partition of $\mathcal{Z}$ into disjoint subsets $\mathcal{K}=\left\{\mathcal{Z}_{1}, \ldots, \mathcal{Z}_{k}\right\} \subseteq \mathcal{Z}$ (some of which may be empty), minimising the overall sum of squared distances

$$
\mathcal{D}^{k}(\mathcal{K})=\sum_{j=1}^{k} \sigma^{2}\left(\bar{S}_{j}, \mathcal{Z}_{j}\right),
$$

where $\bar{S}_{j}$ is the empirical Fréchet mean of the cluster $\mathcal{Z}_{j} \subseteq \mathcal{Z}$. The $k$-means objective can be reinterpreted in terms of finding the centroids $\bar{S}_{j}$. That is, we search for $k$ (possibly nondistinct) centroids minimising the nearest neighbour dispersion (2), where $\mathcal{Z}_{j}$ contains the points with nearest centroid $\bar{S}_{j}$. Both phrasings - finding the centroids or finding the partition - are equivalent, since the Fréchet mean is the dispersion minimising centroid (1).

\section{B. Symmetric Positive Definite Matrices}

A Symmetric Positive Definite (SPD) matrix $S$ is a square symmetric matrix with real entries satisfying $x^{T} S x>0$ for all vectors in $x \in \mathbb{R}^{m} \backslash\{\mathbf{0}\}$, where $S$ is $m \times m$. Equivalently, a square symmetric matrix is positive definite if all of its eigenvalues are positive. SPD matrices can be understood geometrically as encoding ellipsoids, or scaling along a set of $m$ orthonormal basis vectors in $\mathbb{R}^{m}$. That is, $S$ can be decomposed as $S=U^{T} D U$, where $U$ is orthogonal and $D$ is diagonal. In particular, this implies a simple expression [4] for calculating an arbitrary analytic matrix function $f$, such as the matrix logarithm Log, or exponential Exp, as
$\mathbf{f}(S)=U^{T} \mathbf{f}(D) U$ where $\mathbf{f}(D)$ is the diagonal matrix with $i$ th diagonal entry $\mathbf{f}(D)_{i i}=\mathbf{f}\left(D_{i i}\right)$.

The space $\mathbb{S}_{m}$ of SPD matrices is closed under addition and multiplication by positive real numbers, but not under multiplication by non-negative real numbers, thus forming the interior of a blunt convex cone [1]. However, every $S \in \mathbb{S}_{m}$ permits a Cholesky decomposition $S=L L^{T}$, where $L$ is a lower triangular matrix with positive real diagonals [4], and in Section II-A we use the Cholesky decomposition to construct a diffeomorphism between $\mathbb{S}_{m}$ and $\mathbb{R}^{m(m+1) / 2}$.

\section{LOG-CHOLESKY $k$-MEANS}

\section{A. Log-Cholesky distance and mean}

We use $\mathbb{L}_{m}$ to denote the space of lower triangular matrices with positive diagonal. The map $\mathscr{L}: \mathbb{S}_{m} \rightarrow \mathbb{L}_{m}$, that sends an SPD matrix to its Cholesky factor, was shown in [4. Proposition 2] to be a diffeomorphism. So, with this oneto-one correspondence in mind, define for $L, K \in \mathbb{L}_{m}$ the distance function,

$$
\begin{aligned}
d_{C}^{2}(L, K) & =\|\lfloor L\rfloor-\lfloor K\rfloor\|_{F}^{2} \\
& +\|\log (\mathbf{D}(L))-\mathbf{L o g}(\mathbf{D}(K))\|_{F}^{2},
\end{aligned}
$$

where $\|\cdot\|_{F}$ is the Frobenius (i.e., vectorised Euclidean) norm and $\mathbf{D}$ is the diagonalisation function that maps off diagonal elements to 0. In [4. Proposition 10] it was also shown that, under this distance function, the Fréchet mean of a random SPD matrix $S$ exists, is unique, and takes the following closed form, provided that $\mathbb{E} d_{C}^{2}(L, \mathscr{L} S)<\infty$ for some $L \in \mathbb{L}_{m}$.

$$
\mathbb{E} S=\mathscr{L}^{-1}[\mathbb{E}\lfloor\mathscr{L} S\rfloor+\mathbf{E x p}\{\mathbb{E} \log (\mathbf{D}(\mathscr{L} S)\}] .
$$

It follows [4. Corollary 12] that a subset $\mathcal{Z} \subseteq \mathbb{S}_{m}$ has empirical Fréchet mean $\bar{S}$ given by inverting

$$
\mathscr{L}(\bar{S})=\sum_{S \in \mathcal{Z}} \frac{\lfloor\mathscr{L} S\rfloor}{|\mathcal{Z}|}+\mathbf{E x p}\left\{\sum_{S \in \mathcal{Z}} \frac{\log (\mathbf{D}(\mathscr{L} S))}{|\mathcal{Z}|}\right\} .
$$

\section{B. Reduction to Euclidean mean}

Define the map $\mathcal{V}: \mathbb{S}_{m} \rightarrow \mathbb{R}^{m(m+1) / 2}$ given by

$$
\mathcal{V}(S)=\left(\ell_{1}, \ldots, \ell_{m(m-1) / 2}, d_{1}, \ldots, d_{m}\right)^{T},
$$

where $d_{i}=\log \left(\mathscr{L} S_{i i}\right)$, the $\log$ transformed $i$ th diagonal element of $\mathscr{L} S$, and $\ell_{i}=(\operatorname{vec}\lfloor\mathscr{L} S\rfloor)_{i}$, the $i$ th coordinate output of the vectorisation operator, applied to the lower triangle of $\mathscr{L} S$. So, $\mathcal{V}$ is a composition of the Cholesky map $\mathscr{L}$, the scalar logarithm, and a vectorisation that drops the (vanishing) upper triangle elements. Hence, $\mathcal{V}$ is bijective and continuous, since $\mathscr{L}$ is a diffeomorphism from $\mathbb{S}_{m}$ to $\mathbb{L}_{m}$. It follows that $\mathcal{V}$ preserves all compact sets. Furthermore, we now have that (3) reduces to the Euclidean distance. So, (5) can be written,

$$
\bar{S}=\mathcal{V}^{-1}\left(\frac{1}{|\mathcal{Z}|} \sum_{S \in \mathcal{Z}} \mathcal{V}(S)\right)
$$

In other words, $\mathcal{V}(S)$ provides a one-to-one continuous mapping between the space of SPD matrices and a Euclidean 
space, where the Log-Cholesky Fréchet mean reduces to the Euclidean mean. It follows that the corresponding Fréchet $k$ means is exactly the same as a Euclidean $k$-means.

\section{Theoretical results}

For fixed $k$, we can write the $k$-means objective function for $n$ observations $S_{i}, i \in[n]$, as

$$
\mathcal{D}_{n}^{k}\left(X^{k}\right)=\frac{1}{n} \sum_{i=1}^{n} \min _{j \in[k]}\left\|\mathcal{V}\left(S_{i}\right)-X_{j}\right\|^{2},
$$

where $X^{k}=\left\{X_{1}, \ldots, X_{k}\right\}$, and $X_{j} \in \mathbb{R}^{m(m+1) / 2}$, for each $j \in[m]$. Let

$$
X_{n}^{k}=\left\{X_{n, 1}^{k}, \ldots, X_{n, k}^{k}\right\}=\underset{X}{\arg \min } \mathcal{D}_{n}^{k}\left(X^{k}\right) ;
$$

then, with some abuse of notation, the set of optimal cluster centers in $\mathbb{S}_{m}$ is:

$$
\mathcal{V}^{-1}\left(X_{n}^{k}\right)=\left\{\mathcal{V}^{-1}\left(X_{n, 1}^{k}\right), \ldots, \mathcal{V}^{-1}\left(X_{n, k}^{k}\right)\right\}
$$

Let $P$ be a probability measure on the set $\mathbb{S}_{m}$, and let $A$ be a finite subset of $\mathbb{R}^{m(m+1) / 2}$. Further, define

$$
\mathcal{Q}(A, P)=\mathbb{E}_{P_{S}}\left[\min _{\alpha \in A}\left\|\mathcal{V}\left(S_{i}\right)-\alpha\right\|^{2}\right]
$$

and $m_{k}(P)=\inf \{\mathcal{Q}(A, P): \#(A) \leq k\}$. The following consistency theorem can be obtained via the main theorem of [17].

Proposition 1. Assume that $S_{1}, \ldots, S_{n}$ are IID and arise from a data generating process with probability measure $P_{S}$, with $\mathbb{E}_{P_{S}}\|\mathcal{V}(S)\|^{2}<\infty$, and that for each $j \in[k]$, there exists a unique set $A^{j}$, such that $\mathcal{Q}\left(A^{j}, P_{S}\right)=m_{j}\left(P_{S}\right)$. Then, $X_{n}^{k} \rightarrow A^{k}$ and $\mathcal{D}_{n}^{k}\left(X^{k}\right) \rightarrow m_{k}\left(P_{S}\right)$, almost surely.

Let $\underline{X}_{n}^{k}$ and $\underline{A}^{k}$ be vectors containing the elements of $X_{n}^{k}$ and $A^{k}$, respectively, and let $\underline{\mathcal{Q}}\left(\underline{A}^{k}, P\right)$ be a vectorinput version of $\mathcal{Q}\left(A^{k}, P\right)$. Further, denote the Hessian of $\underline{\mathcal{Q}}$, with respect to $\underline{A}^{k}$, by $\mathbb{H}\left[\underline{\mathcal{Q}}\left(\underline{A}^{k}, P\right)\right]$. We can deduce the asymptotic normality result regarding $\underline{X}_{n}^{k}$ via the main theorem of [10].

Proposition 2. In addition to the conditions of Proposition 1 , assume that $P_{S}$ can be characterized by a probability density function $f_{\mathcal{V}}$, with respect to the transformation $\mathcal{V}(S) \in \mathbb{R}^{m(m+1) / 2}$, where $f_{\mathcal{V}}(S) \leq h(\|\mathcal{V}(S)\|)$, for all $S \in \mathbb{S}_{m}$, such that $\int_{0}^{\infty} r^{m(m+1) / 2} h(r) d r$, for some dominating function $h$. If we further assume that $\mathbb{H}\left[\underline{\mathcal{Q}}\left(\underline{A}^{k}, P_{S}\right)\right]$ is positive definite, then $n^{-1 / 2}\left(\underline{X}_{n}^{k}-\underline{A}_{k}\right)$ is asymptotically normal with mean 0 and covariance $\left[\mathbb{H}\left[\underline{\mathcal{Q}}\left(\underline{A}^{k}, P_{S}\right)\right]\right]^{-1} \Sigma\left[\mathbb{H}\left[\underline{\mathcal{Q}}\left(\underline{A}^{k}, P_{S}\right)\right]\right]^{-1}$, where $\Sigma$ is a $k m(m+1) / 2 \times k m(m+1) / 2$ block diagonal matrix with jth block

$\Sigma_{j}=4 \mathbb{E}_{P_{S}}\left[\mathbf{1}\left\{\mathcal{V}(S) \in M_{j}\right\}\left(\mathcal{V}(S)-\underline{A}_{j}^{k}\right)\left(\mathcal{V}(S)-\underline{A}_{j}^{k}\right)^{T}\right]$,

and $M_{j}=\left\{M \in \mathbb{R}^{m(m+1) / 2}: j=\arg \min _{j \in[k]}\left\|M-\underline{A}_{l}^{k}\right\|\right\}$.
Under general assumptions regarding $P_{S}$, Proposition 1 provides the almost sure convergence between $\mathcal{D}_{n}^{k}\left(X^{k}\right)$ and $m_{k}\left(P_{S}\right)$. However, a compactness assumption on the sample space of $S_{1}, \ldots, S_{n}$ allows for the quantification of rates, via the application of Theorems 4-6 of [18] to establish the following result.

Proposition 3. In addition to the conditions of Proposition 1, assume that $P_{S}$ is compactly supported on $\left\{S \in \mathbb{S}_{m}:\|\mathcal{V}(S)\|^{2} \leq r\right\}$ for some $r>0$. Then:

$$
\begin{gathered}
\mathbb{E}_{P_{S}} \mathcal{Q}\left(X_{n}^{k}, P_{S}\right)-m_{k}\left(P_{S}\right) \leq C_{1} n^{-1 / 2}, \\
m_{k}\left(P_{S}\right)-\mathbb{E}_{P_{S}} \mathcal{D}_{n}^{k}\left(X_{n}^{k}\right) \leq C_{2} n^{-1 / 2}, \text { and } \\
\mathbb{E}_{P_{S}} \mathcal{Q}\left(X_{n}^{k}, P_{S}\right)-m_{k}\left(P_{S}\right)=O\left(n^{-1 / 2} \log ^{1 / 2} n\right),
\end{gathered}
$$

almost surely, where $C_{1}$ and $C_{2}$ are constants that only depend on $m, k$, and $r$.

Thus far, we have assumed that $k$ is known. However, for unknown $k$, we require a procedure that estimates its value. Let $k \in \mathbb{K} \subset \mathbb{N}$ and define,

$$
k^{*}=\min _{k \in \mathbb{K}} m_{j}(P)
$$

for some $P$. We can estimate $k^{*}$ using the Bayesian information criterion (BIC) inspired estimator:

$$
k_{n}^{*}=\min _{k \in \mathbb{K}} \mathcal{D}_{n}^{k}\left(X_{n}^{k}\right)+m(m+1) \frac{k \log n}{n} .
$$

Via Theorem 8.1 and Corollary 8.2 of $[19]$, we have the following result.

Proposition 4. Assume the conditions of Proposition 3. If $\mathbb{H}\left[\underline{\mathcal{Q}}\left(\underline{A}^{k}, P_{S}\right)\right]$ is positive define for each $k \in \mathbb{K} \subset \mathbb{N}$, then $\lim _{n \rightarrow \infty} \operatorname{Pr}\left(k_{n}^{*} \neq k^{*}\right)=0$.

\section{APPLICATION}

In flat and arid regions of Australia, the high evaporation rates often imply the absence of surface water storage available for human consumption, irrigation, or mining. In most cases, it results in an increased dependence on groundwater, in overextraction of groundwater resources, and in groundwater level decrease. Depending on the aquifer conditions, it decreases groundwater discharge into streams and limits the availability of shallow groundwater resources for the GroundwaterDependant Ecosystems/Vegetation (GDE/V) [20]. While it is crucial to monitor GDV health where groundwater resources are exploited, large-scale mapping techniques [21] are not multi-temporal, mostly because clouds limit the coverage of the input imagery products (multispectral) during the wetter months. Cloud-insensitive Synthetic Aperture Radar (SAR) data offer an opportunity for monitoring GDEs [14] but further research is required to better extract the GDV information it contains.

From SAR data, both intensity and coherence products potentially contain information about GDV: The like-polarised band $\mathrm{VV}$, dominated by double-bounce and soil-interaction 
scattering mechanisms (i); the cross-polarised band VH, representing mostly the volumetric scattering and other angularshifts during signal bounce (ii); and the InSAR coherence matrix $\mathrm{CC}$, derived by comparing the phase of two likepolarised bands (VV) of two subsequent acquisitions (repeat path) (iii).

GDVs are expected to have a stable canopy over time as compared to non-GDVs, due to their ability to supplement their water requirements using groundwater during times of water deficit and drought. As such, the proportions of volumetric, soil, and double-bounce scattering mechanisms are expected to be relatively stable in time. In [14], an effort is made to leverage this behaviour for classification of GDVs from SAR images, on a pixel-by-pixel basis, where the $\mathrm{SARGDE}_{v 1}$ index of a pixel is introduced as

$$
\operatorname{SARGDE}_{v 1}=1 /\left(\sigma_{c c} \sigma_{v h} \mu_{c c}\right) .
$$

Here, $\sigma_{c c}$ and $\mu_{c c}$ are the standard deviation and mean of InSAR coherence $\mathrm{CC}$ for the given pixel, sampled over time, and $\sigma_{v h}$ is the corresponding standard deviation in the linearlyprojected VH band intensity values. A hypothesis in [14] is that a threshold $T$ exists such that GDV locations correspond to pixels with $\operatorname{SARGDE}_{v 1}>T$. To examine this hypothesis, a ground truth of GDV locations is provided in the Bureau of Meteorology GDE atlas [22].

In the present study, we step back from direct GDV classification, and focus on exploratory data mining, to better understand the classification task and $\mathrm{SARGDE}_{v 1}$ behaviour.

\section{A. Methodology}

Each pixel is represented by a multivariate time-series (of 30 observations in each of the VV and $\mathrm{VH}$ bands, and 29 observations in the CC product), acquired over one year, in the Mount Gambier region of Australia, in 2017. At the $\approx 30 \mathrm{~m}$ resolution, the image dimensions are $2044 \times 1433$, so that the number of time-series (pixels) in the sample is $n=2,929,052$. We describe the data products in detail in Section III-B From these products, the sample finite $\ell$-lag autocovariance matrices are computed and transformed to the log-Cholesky space via (6). Prior to this transformation, downsampling is performed via local averaging within $p \times p$ patches, to decrease variability. The patch size, $p$, and lag length, $\ell$, are hyperparameters that we choose by maximising the adjusted Rand index [23] for the agreement between $k$-means clusters and ground truth locations.

Given the obtained hyperparameters, an optimal number of clusters $k_{n}^{*}$ are chosen via the BIC inspired estimator 8. We then seek to further justify this choice, by estimating the $\operatorname{SARGDE}_{v 1}$ variability explained by the $k_{n}^{*}$ clusters, via $k_{n}^{*}$-way ANOVA. We compare this to a 2-way ANOVA with simplifying restriction $k=2$, to produce an estimate of the additional variability explained by the $k_{n}^{*}>2$ clusters.

Finally, we visualise, in a log-Cholesky space, the pixels that belong to $k$-means clusters that have more than $5 \%$ empirical probability of overlap with GDV. In the same space, we visualise the sample quartiles of $\mathrm{SARGDE}_{v 1}$. From this, we draw conclusions about $\mathrm{SARGDE}_{v 1}$, and make suggestions for future efforts to classify GDV.

For repeatability, all scripts (in the $\mathrm{R}$ programming language) and pre-processed data are available in an online repository at [24].

\section{B. Data pre-processing}

A total of 30 temporally consecutive Sentinel-1A Interferometric Wide (IW) images in Single-Look-Complex (SLC) format were downloaded via the Alaskan Satellite Facility (ASF) web portal [25] and processed similarly to [14]. The time-series consist of images acquired along the same orbital track and Line-Of-Sight (LOS) angle, which facilitates the interpretation of SAR intensity change over time (i.e., no significant LOS change to take into account or compensate for) and allows the creation of Interferometric SAR (InSAR) coherence maps [26]. The 12-day repeat path of Sentinel satellites guarantees $\approx 30$ intensity images per year, and $\approx 29$ coherence maps per year.

Sentinel-1 IW images were processed using SARscape 5.5 [27]. The images are multi-looked (i.e., decrease in resolution) with a factor $8 / 2$ in Range/Azimuth to produce a regular matrix grid at $\approx 30 \mathrm{~m}$ resolution. This reduces data size and granular noise (speckle) inherent to SAR data. All images were co-registered and spatio-temporally filtered [28] to remove residual noise. Images are then calibrated and converted into backscatter coefficients following a Gamma Nought calibration (correction for local incidence angle variations using the ALOS-3D Digital Elevation Model [29]), and projected along a linear scale. Coherence matrices are computed at the same resolution as the intensity matrices $(30 \mathrm{~m})$ and with a $5 \times 5$ pixel analysis window. They were produced in a time-line process, where each coherence matrix is produced by matching with the subsequent image.

\section{Results}

As shown in Figure 1, the hyperparameters maximising the adjusted Rand index $R$, for agreement of $k$-means classes with GDV locations, were $\ell=1$ and $p=9$. Note that the spike in $R$, seen in Figure 1 near $k=2$, occured only in the CC product, while $\mathrm{VV}$ and $\mathrm{VH}$ showed very low overlap with GDV. We expect, a priori, that the $\mathrm{CC}$ product carries the majority of accessible information about vegetation [14], and this result appears to confirm this expectation. Furthermore, it is feasible that the observed decrease in $R$, for $k>2$, in the $\mathrm{CC}$ product, is due to GDV qualities being split between multiple $k$-means classes. From this point on, we focus our analysis on the $\mathrm{CC}$ product.

The BIC inspired estimator [8, with the obtained hyperparameters, gave $k_{n}^{*}=15$ when explored over $k \in[50]$. For the corresponding 15-way ANOVA, the adjusted coefficient of multiple correlation was 0.532 , indicating that $\approx 53 \%$ of the variability in $\mathrm{SARGDE}_{v 1}$ is explained by the 15 clusters. For comparison, only $\approx 20 \%$ of the variability is explained when $k=2$ (that is, when fitting $k$-means with only 2 clusters). This $33 \%$ increase in explained $\mathrm{SARGDE}_{v 1}$ variability further 

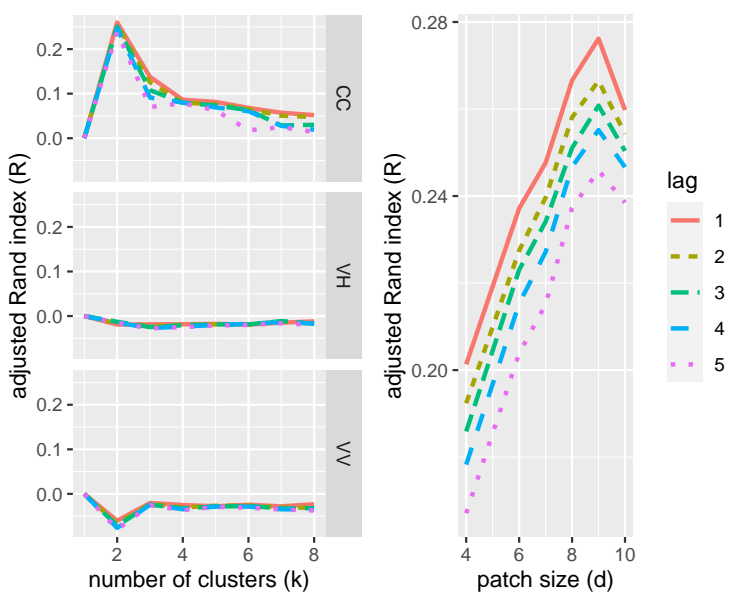

Figure 1. The left panel gives the adjusted Rand index for lags $\ell \in[5]$, clusters $k \in[8]$ and each of the products $\mathrm{CC}, \mathrm{VH}$ and $\mathrm{VV}$. The right panel gives that for $k=2$, in the CC product, and patch sizes $p \in\{4,5, \ldots, 10\}$.

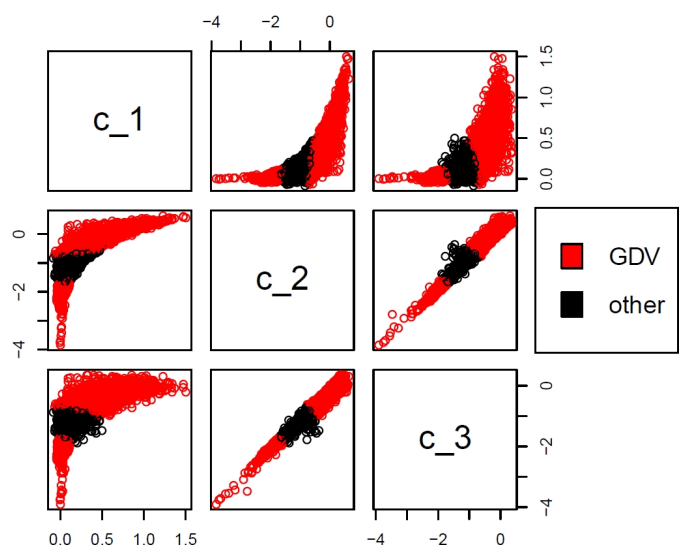

Figure 2. Cross-sectional projection scatter plots of the $n$ time-series (pixels), represented in the 3-dimensional space of autocovariance matrices transformed via (6). Pixels in $k$-means clusters $1,2,5$ and 12 (clusters with greater than $5 \%$ empirical probability of GDV) are coloured black, and all others are red.

justifies the use of $k>2$, at least for the purpose of understanding SARGDE S $_{v 1}$.

Of the $15 k$-means classes, only four have more than $5 \%$ of pixels overlapping with GDV. These are clusters 1,2,5 and 12. In Figure 2 these four clusters are coloured black, and the remaining are coloured red. Time-series (pixels) are represented in Figure 2 by the transformation (6) of their autocovariance matrices. Notice that the 4 classes are neighbours, and occupy a band near the middle of the larger cluster. Pixels outside of this band have a low (less than 5\%) proportion of overlap with GDV.

For comparison with the $\mathrm{SARGDE}_{v 1}$ quartiles, Figure 3 colours the lower $25 \%$ quartile black, the middle $50 \%$ red, and the upper $25 \%$ green. We see that high $\mathrm{SARGDE}_{v 1}$ pixels occupy a band near the middle of the larger cluster (green in Figure 3, but that this band is wide enough that it appears to transgress into the regions with less than $5 \%$ empirical

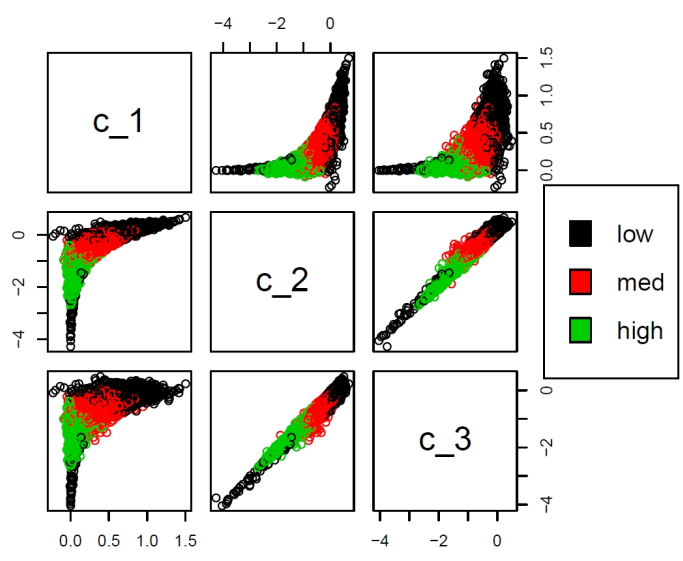

Figure 3. Cross-sectional projection scatter plots of the $n$ time-series (pixels), represented in the 3-dimensional space of autocovariance matrices transformed via 6. Pixels whose $\mathrm{SARGDE}_{v 1}$ is in the lower $25 \%$ sample quartile are coloured black, the middle $50 \%$ red, and the upper $25 \%$ green.

probability of GDV (red, in Figure 2).

\section{DISCUSSION}

The observation that the highest $\mathrm{SARGDE}_{v 1}$ index values transgress into regions of low empirical probability of GDV, suggests a potentially better approach than using a single threshold, $T$, above which SARGDE $_{v 1}$ classifies pixels as GDV. Instead, a lower threshold $T_{\ell}$ and upper threshold $T_{u}$, may be sought, providing an optimal $\mathrm{SARGDE}_{v 1}$ interval, in terms of GDV classification performance. Visually, we observe in Figure 4, that higher values of $\operatorname{SARGDE}_{v 1}$ do not necessarily correspond more to GDV. This is our suggestion for future efforts to improve on SARGDE $\mathrm{S}_{v 1}$. Alternatively, a classifier can be constructed from the $k$-means clusters, directly, instead of using the raw $\mathrm{SARGDE}_{v 1}$ values.

\section{A. Future work}

- We have treated the autocovariance matrices of timeseries (pixels) as stationary, though more information may be obtained by capturing dynamics via covariance trajectories in the space of SPD matrices. See, e.g., [30].

- Due to the one-to-one mapping of any SPD matrix to a real vector, via a differentiable transformation, one can endow the space of SPD matrices with any distribution on multivariate real numbers, such as the Gaussian distribution, and obtain a distribution over the SPD vector via a transformation of variables construction. This then allows for the conduct of model-based clustering, via the methods of [31].

- A similar construction to (6) exists for the log-Euclidean metric norm, in which the off diagonals are mapped to twice their value. Thus, we expect similar properties to those proved in Section 6 to hold.

- This approach to clustering has not made use of information regarding the spatial dependence between pixels across patches. A variety of methods exist that may make 

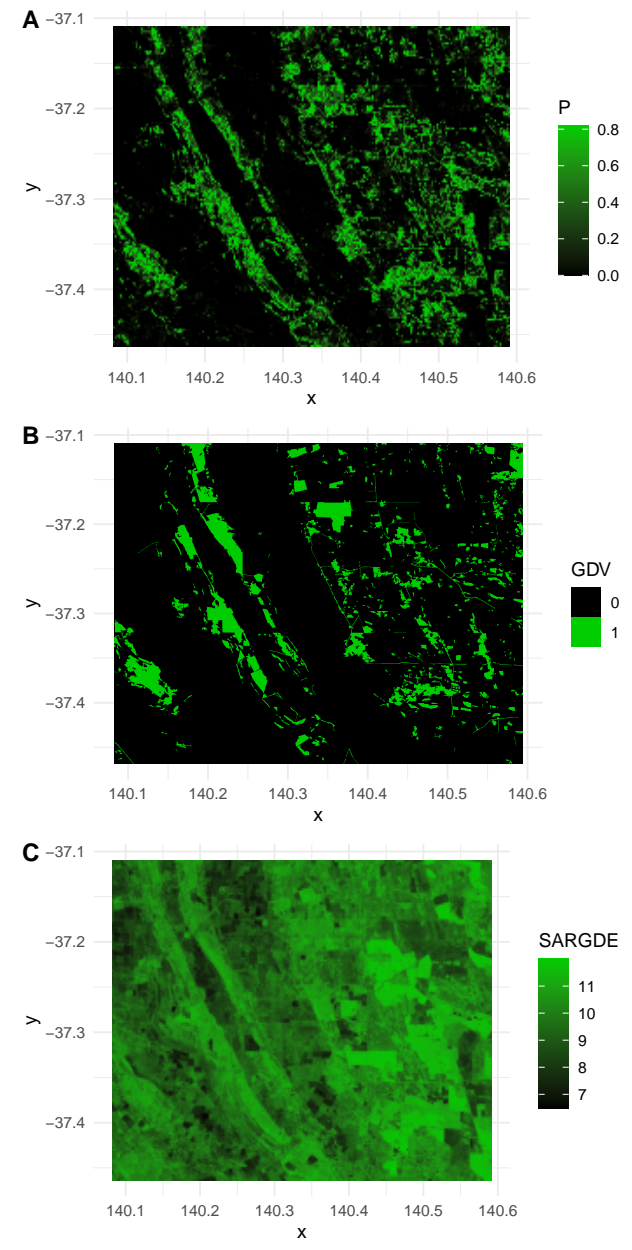

Figure 4. (A) The $15 k$-means classes, coloured by empirical probability $P$ of overlap with GDV; (B) the Bureau of Meteorology GDV atlas ground truth labels; (C) the $\mathrm{SARGDE}_{v 1}$ index.

use of this information, such as, for example, spatial smoothing via Markov random fields.

\section{REFERENCES}

[1] X. Pennec, S. Sommer, and T. Fletcher, Riemannian Geometric Statistics in Medical Image Analysis. Academic Press, 2019.

[2] S. Aghabozorgi, A. S. Shirkhorshidi, and T. Y. Wah, "Time-series clustering-a decade review," Information Systems, vol. 53, pp. 16-38, 2015.

[3] M. Dai, Z. Zhang, and A. Srivastava, "Analyzing dynamical brain functional connectivity as trajectories on space of covariance matrices," IEEE transactions on medical imaging, vol. 39, no. 3, pp. 611-620, 2019.

[4] Z. Lin, "Riemannian geometry of symmetric positive definite matrices via cholesky decomposition," SIAM Journal on Matrix Analysis and Applications, vol. 40, no. 4, pp. 1353-1370, 2019.

[5] X. Pennec, P. Fillard, and N. Ayache, "A Riemannian framework for tensor computing," International Journal of computer vision, vol. 66, no. 1, pp. 41-66, 2006.

[6] M. Calvo and J. M. Oller, "An explicit solution of information geodesic equations for the multivariate normal model," Statistics \& Risk Modeling, vol. 9, no. 1-2, pp. 119-138, 1991.

[7] T. Zhang, "A majorization-minimization algorithm for computing the Karcher mean of positive definite matrices," SIAM Journal on Matrix Analysis and Applications, vol. 38, no. 2, pp. 387-400, 2017.
[8] V. Arsigny, P. Fillard, X. Pennec, and N. Ayache, "Geometric means in a novel vector space structure on symmetric positive-definite matrices," SIAM journal on matrix analysis and applications, vol. 29, no. 1, pp. 328-347, 2007.

[9] M. Faraki, M. Palhang, and C. Sanderson, "Log-Euclidean bag of words for human action recognition," IET Computer Vision, vol. 9, no. 3, pp. 331-339, 2014

[10] D. Pollard, "A central limit theorem for k-means clustering," The Annals of Probability, vol. 10, no. 4, pp. 919-926, 1982.

[11] _ , "Strong consistency of k-means clustering," The Annals of Statistics, pp. 135-140, 1981.

[12] R. Jin, A. Goswami, and G. Agrawal, "Fast and exact out-of-core and distributed k-means clustering," Knowledge and Information Systems, vol. 10, no. 1, pp. 17-40, 2006.

[13] H. Lee, H.-J. Ahn, K.-R. Kim, P. T. Kim, and J.-Y. Koo, "Geodesic clustering for covariance matrices," Communications for Statistical Applications and Methods, vol. 22, no. 4, pp. 321-331, 2015.

[14] P. Castellazzi, T. Doody, and L. Peeters, "Towards monitoring groundwater-dependent ecosystems using synthetic aperture radar imagery," Hydrological Processes, vol. 33, no. 25, pp. 3239-3250, 2019.

[15] M. Arnaudon, F. Barbaresco, and L. Yang, "Medians and means in Riemannian geometry: existence, uniqueness and computation," in Matrix Information Geometry. Springer, 2013, pp. 169-197.

[16] X. Pennec, "Probabilities and statistics on Riemannian manifolds: Basic tools for geometric measurements." in NSIP, vol. 3. Citeseer, 1999, pp. 194-198.

[17] D. Pollard, Convergence of stochastic processes. Springer-Verlag New York, 1984.

[18] T. Linder, "Learning-theoretic methods in vector quantization," in Principles of nonparametric learning. Springer, 2002, pp. 163-210.

[19] J.-P. Baudry et al., "Estimation and model selection for model-based clustering with the conditional classification likelihood," Electronic journal of statistics, vol. 9, no. 1, pp. 1041-1077, 2015.

[20] S. Richardson, E. Irvine, R. Froend, P. Boon, S. Barber, and B. Bonneville, "Australian groundwater-dependent ecosystem toolbox. part 1: Assessment framework," Waterlines report, National Water Commission, Canberra, 2011.

[21] T. M. Doody, O. V. Barron, K. Dowsley, I. Emelyanova, J. Fawcett, I. C. Overton, J. L. Pritchard, A. I. Van Dijk, and G. Warren, "Continental mapping of groundwater dependent ecosystems: A methodological framework to integrate diverse data and expert opinion," Journal of Hydrology: Regional Studies, vol. 10, pp. 61-81, 2017.

[22] (2020) Bureau of meteorology, gde atlas. [Online]. Available: http://www.bom.gov.au/water/groundwater/gde/map.shtml

[23] L. Hubert and P. Arabie, "Comparing partitions," Journal of classification, vol. 2, no. 1, pp. 193-218, 1985

[24] D. Fryer. (2020) kmspd: $k$-means with symmetric positive definite matrices in R. [Online]. Available: https://github.com/frycast/kmspd

[25] (2020) Alaskan satellite facility. [Online]. Available: https://vertex.daac. asf.alaska.edu

[26] H. A. Zebker, J. Villasenor et al., "Decorrelation in interferometric radar echoes," IEEE Transactions on geoscience and remote sensing, vol. 30 , no. 5, pp. 950-959, 1992

[27] Envi sarscape version 5.5. [Online]. Available: https://www. harrisgeospatial.com/Software-Technology/ENVI

[28] G. De Grandi, M. Leysen, J. Lee, and D. Schuler, "Radar reflectivity estimation using multiple sar scenes of the same target: technique and applications," in IGARSS'97. 1997 IEEE International Geoscience and Remote Sensing Symposium Proceedings. Remote Sensing-A Scientific Vision for Sustainable Development, vol. 2. IEEE, 1997, pp. 10471050.

[29] J. Takaku, T. Tadono, and K. Tsutsui, "Generation of high resolution global dsm from ALOS prism." ISPRS Annals of Photogrammetry, Remote Sensing \& Spatial Information Sciences, vol. 2, no. 4, 2014.

[30] Z. Zhang, J. Su, E. Klassen, H. Le, and A. Srivastava, "Rate-invariant analysis of covariance trajectories," Journal of Mathematical Imaging and Vision, vol. 60, no. 8, pp. 1306-1323, 2018.

[31] G. J. McLachlan and D. Peel, Finite mixture models. John Wiley \& Sons, 2004. 